\title{
Distributed Detection in OFDM based Ad Hoc Overlay Systems
}

\author{
Ulrich Berthold, Friedrich K. Jondral \\ Universität Karlsruhe (TH), Germany \\ Institut für Nachrichtentechnik \\ berthold@int.uni-karlsruhe.de
}

\begin{abstract}
Overlay systems are a feasible approach to increase the efficiency of spectrum use. An independent secondary user (SU) system operates in the same frequency band as a primary user (PU) system. In order to avoid mutual interference, an important task of every SU system is to periodically monitor the PU system's allocation. In this paper, we investigate the question how many SUs have to be deployed in the system area of an ad hoc network in order to meet a given network wide detection probability and how this effects the false alarm probability (and thus the performance of the SU system). This is achieved with the help of geometric random graph theory and results from a distributed detection approach. In our investigations we consider a scenario with an infinite system area, and therefore neglecting border effects, as well as a scenario including border effects.
\end{abstract}

\section{INTRODUCTION}

With the growing market for mobile communications also the demand for its main technological resource, suitable spectrum, is steadily increasing. Since this resource is naturally limited by its physical properties, an efficient spectrum use gains importance. Motivated by the apparent spectrum scarcity when looking at the frequency plans of regulatory bodies, spectrum measurements have been performed, revealing that large parts of the spectrum are only used during a low percentage of time [1].

There are several approaches for increasing overall spectral efficiency. Within a system, the throughput for a given bandwidth can be optimized on the physical layer by using efficient transmission technologies, e.g., OFDM (orthogonal frequency division multiplex) or MIMO (multiple-input and multiple-output). On a system level, the efficiency of spectrum use can be increased by exploiting the spectrum holes in the time-frequency plane for additional transmissions. This can be done in a cooperative way by the same operator, as, e.g., in GPRS (general packet radio service) where the spare slots (i. e. slots not used for voice calls) are used for data transmissions.

A more general approach is DSA (dynamic spectrum access) which also contains the concept of overlay systems. In an overlay scenario a SU system is operating in the same frequency band as a licensed PU system and exploits the left over spectral resources by dynamically hopping into the spectrum holes and using them for additional independent transmissions. There is a wide range of possible applications for overlay systems [2], but their basic required behavior can be summarized by two assumptions [3]:

- The PU system has priority and must not be effected by the secondary user system.

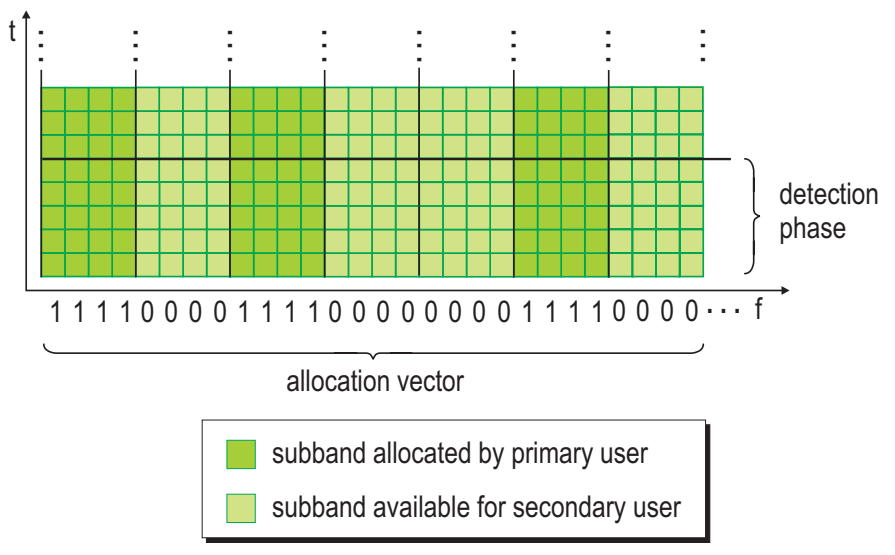

Fig. 1. Allocation of a primary user in the time-frequency plane and the resulting allocation vector.

- The PU system must not be modified.

Therefore, the SU system must periodically perform measurements in order to detect the PU system's current allocation. Since the performance of the detection subsystem effects the PU system as well as the SU system, the requirements are set high. The remainder of this paper is organized as follows. Section II gives a short introduction to OFDM based overlay systems with the focus on ad hoc networks and a brief description of relevant ad hoc properties. In Section III, the network detection probability is defined and upper bounds are derived. Finally, the paper is concluded in Section IV.

\section{OFDM based Overlay Systems in Ad Hoc Mode}

In this paper we focus on SUs based on OFDM, since with OFDM a sophisticated, flexible and efficient technology is available, which has been proven to be feasible for overlay systems [4]. Furthermore, we assume that the PU system is using time/frequency division multiple access (TDMA/FDMA) as access mode. PU systems using code division multiple access (CDMA) are not suitable for an overlay scenario, since the signals are more difficult to detect by the applied energy detector and do not result in spectrum holes in the time/frequency plane. Measurements for detection of PUs can be easily performed without additional hardware, using the already included FFT (fast Fourier transform). Based on the detection results each subcarrier can be activated individually for the SU's transmission, thus dynamically adapting to the 


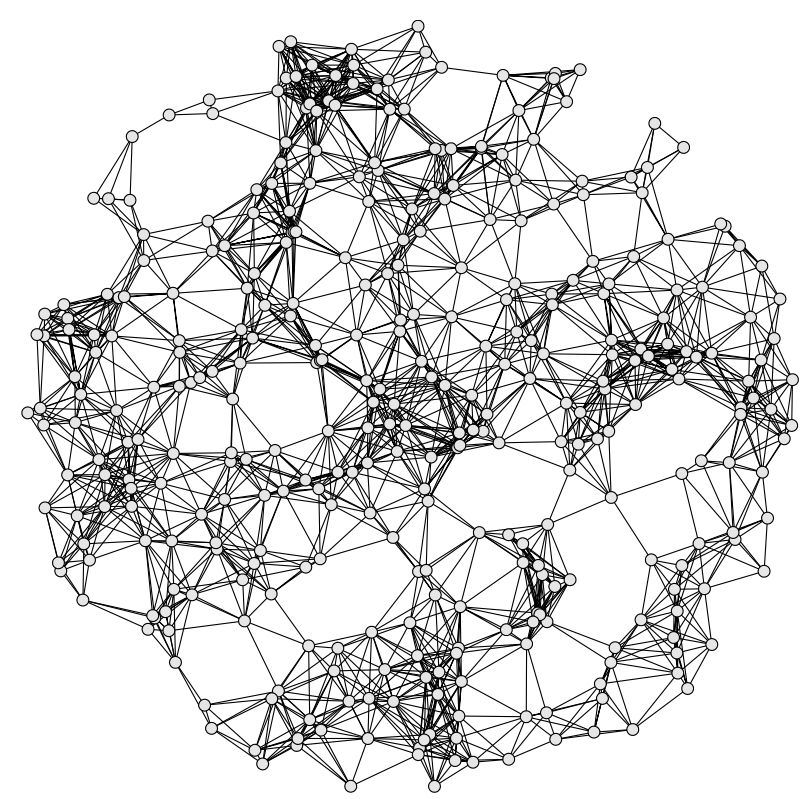

Fig. 2. Ad Hoc network with $N=250$ SUs with a normalized transmission range $\hat{r}_{0}=0.2$.

current PU's allocation and avoiding interference. Fig. 1 shows part of the time frequency plane with an example of the PU's allocation. The bandwidth of each PU's subchannel is filled with four subcarriers of the SU system. Each small box represents an FFT-value. The allocation vector is derived from the measurements, indicating whether a subcarrier is blocked by the PU system ("1") or may be used by the SU system ("0"). Note, that both, the physical (PHY) and medium access layer (MAC), are involved in the detection process. The PHY layer performs the actual measurement whereas the MAC layer is responsible for the coordination and initiation of the detection phases.

We now shift to a scenario where the overlay system operates in ad hoc mode. A possible application could be, e. g., a network of sensors which is deployed in a certain area and which communicates in a frequency band actually dedicated to an independent PU system. We first resume the basic properties of geometric graphs and distributed detection.

\section{A. System Model}

Graph theory is a suitable tool to describe and analyze the properties of ad hoc networks. An ad hoc network consists of $N$ SUs which are distributed in space and are represented by the set of nodes $\mathcal{X}$ with $N=|\mathcal{X}|$. We limit our considerations to the 2-dimensional space $\mathbb{R}^{2}$, so that $\mathcal{X} \subset \mathbb{R}^{2}$. If two SUs are able to establish a communication link, they are connected by an edge in the corresponding graph. In general graph theory each node may be connected with any other node of the network [5]. In contrast, for a wireless ad hoc network the edges are determined by the positions of the SUs and their transmission range. Assuming that all SUs are identically equipped, i. e., they have the same transmission power and thus also the same transmission range, the resulting network topology can be described completely by the positions of the SUs and the systemwide transmission range $r_{0}$ of each SU. Accordingly, an ad hoc network can be represented by an undirected graph $G=G\left(\mathcal{X} ; r_{0}\right)$, in which two nodes $\mathbf{u}, \mathbf{v} \in G$ are connected by an edge if they are within each others transmission range, i. e. $|\mathbf{u}-\mathbf{v}| \leq r_{0}$. To achieve more general results, we investigate random graphs. Graphs in which the positions of the nodes are random and the edges depend on the the SUs' transmission range are also called random geometric graphs [6].

\section{B. Number of Neighbors}

The number of neighbors $D$ of a given node $\mathbf{u} \in G$ in an ad hoc network, also called degree of $\mathbf{u}$, plays an important role for the investigations in section III-B. Therefore, we summarize some relevant results which have been derived in [7].

$D$ depends on the distribution of the nodes in the system area $\mathbf{A}$ with the size $A$. We investigate two different cases. First, we start with the more simple case and neglect border effects, which we then include in a second, more realistic scenario. Since border effects will always degrade but not increase the overall performance regarding distributed detection, the borderless scenario will serve as a benchmark.

1) No border effects: A scenario without border effects can be derived by considering a network with an infinite system area, i. e. $A \rightarrow \infty$, and a constant density $\rho=\frac{N}{A}$, thereby $N \rightarrow \infty$. Under these terms, the number of nodes in every finite subarea follows a Poisson distribution and the number of nodes in disjoint subareas are independent random variables [8], i.e., we have a homogeneous Poisson point process with intensity $\rho$ for the number of nodes. Since $D$ is equivalent to the number of nodes placed in a disk of radius $r_{0}$ centered around $\mathbf{u}, D$ has also a Poisson distribution and the probability that $\mathbf{u}$ has $d$ neighbors is given by

$$
P_{\infty}(D=d)=P_{\infty}(d)=\frac{\nu^{d}}{d !} e^{-\nu}
$$

with $E\{D\}=\nu=\rho \pi r_{0}^{2}$. Due to the homogeneity of the Poisson process, $\nu$ is independent of the location and therefore identical for every node.

2) With border effects: We now assume that $\mathbf{A}$ is a disk with radius $a$ in which the $N$ SUs are distributed. $\hat{r}_{0}=r_{0} / a$ is the normalized transmission range of the nodes and $\hat{r}=r / a$ the normalized radial component of the node position, when using polar coordinates with the origin in the center of $\mathbf{A}$. Fig. 2 shows an example scenario with $N=250$ SUs and $\hat{r}_{0}=0.2$. Let $p_{0}(\mathbf{x})$ be the probability that a second node is placed within the transmission range of a given node $\mathbf{x}$. The probability that $D$ nodes are placed in the transmission range of $\mathbf{x}$ is then given by a binomial distribution:

$$
P(D=d \mid \mathbf{x})=\left(\begin{array}{c}
N-1 \\
d
\end{array}\right) p_{0}(\mathbf{x})^{d}\left(1-p_{0}(\mathbf{x})\right)^{N-d-1} .
$$

Note, that for large $N$ and small $p_{0}(\mathbf{x}), P(d \mid \mathbf{x})$ can be approximated by a Poisson distribution with the location dependent expectation $\nu(\mathbf{x})=(N-1) p_{0}(\mathbf{x})$. The unconditional 
probability for a node's number of neighbors can be derived by integration:

$$
P(D=d)=P(d)=\iint_{\mathbf{A}} P(D=d \mid \mathbf{x}) f_{\mathbf{X}}(\mathbf{x}) \mathrm{d} \mathbf{x} .
$$

In the following, we assume a uniform distribution for $\mathbf{X}$ in the area $\mathbf{A}$, with $\mathbf{X}$ describing the positions of the nodes:

$$
f_{\mathbf{X}}(\mathbf{x})= \begin{cases}\frac{1}{A} & \text { for } \mathbf{x} \in \mathbf{A} \\ 0 & \text { otherwise. }\end{cases}
$$

Nevertheless, due to the bounded system area, $p_{0}(\mathbf{x})$ depends on the location of $\mathbf{x}$. When using polar coordinates, $p_{0}(\mathbf{x})$ depends especially on the radial component, since it determines the distance to the border of the system area [7]:

$p_{0}(\mathbf{x})= \begin{cases}\hat{r}_{0}^{2} & \text { for } 0 \leq \hat{r} \leq 1-\hat{r}_{0} \\ \frac{1}{\pi}\left(\hat{r}_{0}^{2} \arccos \frac{\hat{r}^{2}+\hat{r}_{0}^{2}-1}{2 \hat{r} \hat{r}_{0}}+\right. & \\ \left.\arccos \frac{\hat{r}^{2}-\hat{r}_{0}^{2}+1}{2 \hat{r}}-\frac{1}{2} \sqrt{\zeta}\right) & \text { for } 1-\hat{r}_{0}<\hat{r} \leq 1\end{cases}$

with $\zeta=\left(\hat{r}+\hat{r}_{0}+1\right)\left(-\hat{r}+\hat{r}_{0}+1\right)\left(\hat{r}-\hat{r}_{0}+1\right)\left(\hat{r}+\hat{r}_{0}-1\right)$. Using polar coordinates and inserting (2) and (4) in (3) yields

$$
P(d)=\int_{0}^{1} 2 \hat{r} \cdot\left(\begin{array}{c}
N-1 \\
d
\end{array}\right) p_{0}(\hat{r})^{d}\left(1-p_{0}(\hat{r})\right)^{N-d-1} \mathrm{~d} \hat{r}
$$

for the location independent probability of the number of neighbors.

\section{Distributed Detection in Ad Hoc Networks}

How are the properties of an ad hoc network related to the performance of the detection subsystem? How can a required detection probability be achieved in the context of an ad hoc system? These questions are discussed in the following.

\section{A. The Detector Model}

In this paper we use the detector model proposed in [9]. For each licensed channel the detection phase yields $M$ complex valued samples in the frequency domain, for which it is shown that they are complex zero-mean Gaussian random variables represented by

$$
R(m)=S(m)+N(m) ; m=1,2, \ldots, M .
$$

This results in $2 M$ samples stored in the detectors memory. $M$ depends on the number of subcarriers per licensed channel and the number of performed detection cycles, as denoted in Fig. 1. In (7), $\left\{S(m)=S_{x}(m)+j S_{y}(m)\right\}_{m=1}^{M}$ and $\{N(m)=$ $\left.N_{x}(m)+j N_{y}(m)\right\}_{m=1}^{M}$ represent the signal components in frequency domain resulting from the PU system and noise, respectively. The mean powers of $S(m)$ and $N(m)$ are denoted by $2 \sigma_{S}^{2}$ and $2 \sigma_{N}^{2}$. We assume that $S(m)$ and $N(m) ; m=$ $1,2, \ldots, M$ are independent.

To derive the detection probability and the false alarm probability for the detection of a PU system, first the probability density functions are derived, followed by the application of the Neyman-Pearson strategy [10], resulting in a likelihood ratio test with the threshold $\lambda_{0}$. The exact steps for the calculations are omitted here, but can be found in [11]. Finally, we get a detector that is matched to the signal statistics, with the detection probability

$$
\begin{aligned}
P_{D}= & \frac{1}{2^{M}\left(\sigma_{S}^{2}+\sigma_{N}^{2}\right)^{M}(M-1) !} \cdot \exp \left(-\frac{\lambda_{0}}{2\left(\sigma_{S}^{2}+\sigma_{N}^{2}\right)}\right) \\
& \cdot\left(2\left(\sigma_{S}^{2}+\sigma_{N}^{2}\right) \lambda_{0}^{M-1}\right. \\
& \left.\quad+\sum_{m=1}^{M-1}\left(2\left(\sigma_{S}^{2}+\sigma_{N}^{2}\right)\right)^{m+1} \lambda_{0}^{M-m-1} \prod_{l=1}^{m}(M-l)\right)
\end{aligned}
$$

and the false alarm probability $\left(\sigma_{S}^{2}=0\right.$ in (8))

$$
\begin{aligned}
P_{F}= & \frac{1}{\left(2 \sigma_{N}^{2}\right)^{M}(M-1) !} \exp \left(-\frac{\lambda_{0}}{2 \sigma_{N}^{2}}\right) \\
& \cdot\left(2 \sigma_{N}^{2} \lambda_{0}^{M-1}+\sum_{m=1}^{M-1}\left(2 \sigma_{N}^{2}\right)^{m+1} \lambda_{0}^{M-m-1} \prod_{l=1}^{m}(M-l)\right) .
\end{aligned}
$$

This represents a best-case scenario, so that the resulting receiver operating characteristics are upper bounds for the performance of the detection subsystem.

\section{B. Theoretical Bounds for Distributed Detection in Ad Hoc Networks}

In [12] receiver operating characteristics for the detection of a PU system were derived, relating the false alarm probability $P_{F}$ to the detection probability $P_{D}$ of a single SU. To enhance detection performance, a diversity approach for detection in a cell-based SU system with a central base station was proposed. In a cell, several SUs independently perform a detection and signal their result to the base station. The SU base station combines the distributed detection results and generates a systemwide valid allocation vector which finally is distributed back to all SUs in the cell. The cell's overall false alarm and detection probability

$$
\begin{aligned}
& P_{F}^{C}(L)=1-\left(1-P_{F}\right)^{L} \\
& P_{D}^{C}(L)=1-\left(1-P_{D}\right)^{L}
\end{aligned}
$$

is introduced, where $L$ is the number of SUs participating in the distributed detection.

When transferring this distributed detection approach to an ad hoc scenario, we have to take into account that there is no central processing SU available. Nevertheless, each SU can enhance its detection performance by applying the distributed detection approach based on the single detection results of its neighbors. Since the number of neighbors is random, we define the network detection probabilities $P_{D}^{N_{\infty}}\left(\rho ; r_{0}\right)$ and $P_{D}^{N}\left(N ; r_{0}\right)$, for the borderless scenario and the scenario considering border effects, respectively. We assume that the SU knows the number of its neighbors and that every neighbor contributes to the distributed detection process. Signaling issues are neglected at this point. Therefore, the following investigations are theoretical bounds for the average network detection probabilities. 


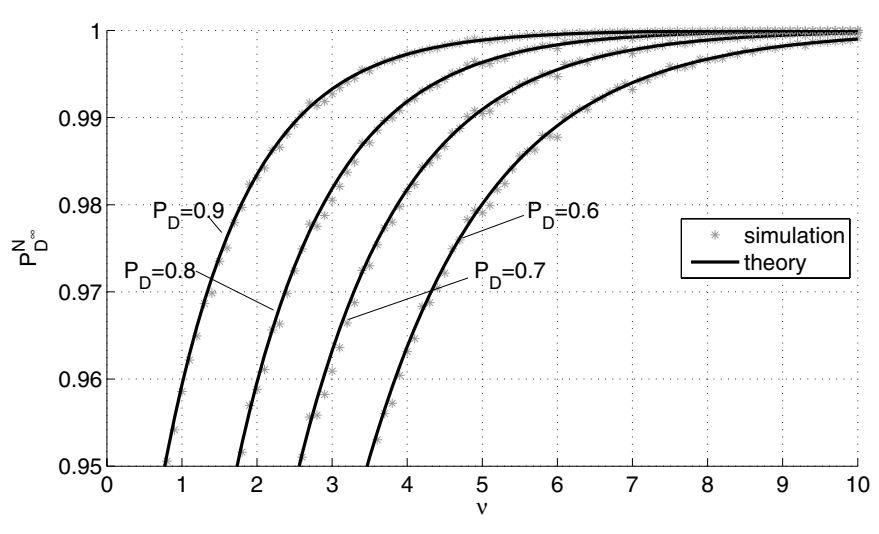

Fig. 3. Network detection probability $P_{D}^{N_{\infty}}$ in the borderless scenario for various single detection probabilities $P_{D}$ depending on the expected number of neighbors $\nu$.

1) No border effects: In the borderless scenario, $P_{D}^{N_{\infty}}\left(\rho ; r_{0}\right)$ depends on the node density and the transmission range, and thus with $\nu=\rho \pi r_{0}^{2}$ also on $\nu$ :

$$
P_{D}^{N_{\infty}}(\nu)=\sum_{d=0}^{\infty} P_{\infty}(d) \cdot P_{D}^{C}(d+1) .
$$

$P_{D}^{C}(d+1)$ is weighted by the probability that a SU has $d$ neighbors. Plugging (1) and the second equation of (10) into (11) and with $L=d+1$ we get

$$
P_{D}^{N_{\infty}}(\nu)=\sum_{d=0}^{\infty} \frac{\nu^{d}}{d !} e^{-\nu} \cdot\left(1-\left(1-P_{D}\right)^{d+1}\right) .
$$

In a similar way, the SU system's network false alarm probability $P_{F}^{N_{\infty}}(\nu)$ can be expressed when using the first equation of (10):

$$
P_{F}^{N_{\infty}}(\nu)=\sum_{d=0}^{\infty} \frac{\nu^{d}}{d !} e^{-\nu} \cdot\left(1-\left(1-P_{F}\right)^{d+1}\right) .
$$

Fig. 3 shows the plots of $P_{D}^{N_{\infty}}(\nu)$ for several single detection probabilities $P_{D}$. For a given network detection probability $P_{D}^{N_{\infty}}=0.999$ and with, e.g., $P_{D}=0.8$ the expected number of neighbors needs to be at least $\nu=6.6$. To simulate the borderless scenario, the SUs were placed randomly in an area larger than the system area, but only the SUs in the system area were considered for the results. The number of each SU's neighbors was determined and then the minimum possible resulting distributed detection probability was calculated, assuming that every neighbor contributes to the distributed detection. The simulation results in Fig. 3 are close to the analytical results.

The effect on the false alarm probability is shown in Table I for $M=8$, SNR $=2 \mathrm{~dB}$ and a given network detection probability $P_{D}^{N_{\infty}}=0.999$. Depending on $\nu$, the required single detection probability for achieving the given network detection probability was calculated with the help of (12). The corresponding single false alarm probability is shown in the third column, from which finally the network false alarm probability $P_{F}^{N_{\infty}}$ is calculated (Eqn. (13)). The
TABLE I

AD HOC NETWORK'S FALSE ALARM PROBABILITY $P_{F}^{N}(\nu)$ $\left(M=8, \mathrm{SNR}=2 \mathrm{~dB}, P_{D}^{N_{\infty}}=0.999\right)$

\begin{tabular}{|c|c|c|c|}
\hline$v$ & $P_{D}$ & $P_{F}$ & $P_{F}^{N_{\infty}}(v)$ \\
\hline 0 & 0.999 & 0.853 & 0.853 \\
\hline 3 & 0.981 & 0.384 & 0.805 \\
\hline 5 & 0.906 & 0.096 & 0.441 \\
\hline 7 & 0.781 & 0.020 & 0.150 \\
\hline 9 & 0.656 & 0.005 & 0.050 \\
19 & 0.344 & $\approx 0$ & 0.002 \\
\hline
\end{tabular}

results in Table I show, that an acceptable performance of the detection subsystem can be achieved, when the node density and transmission range of the ad hoc network result in $\nu \geq 7$.

2) With border effects: When considering border effects, we have a defined number of SUs in a bounded system area. In this case, the resulting network detection probability does not only depend on $\nu$, but on the specific combination of $N$ and $r_{0}$, since $p_{0}$ depends on $r_{0}$, according to (5). Similar to (11), the network detection probability is then given by

$$
P_{D}^{N}\left(N ; r_{0}\right)=\sum_{d=0}^{N-1} P(d) \cdot P_{D}^{C}(d+1),
$$

which yields

$$
\begin{aligned}
P_{D}^{N}\left(N ; r_{0}\right)= & \sum_{d=0}^{N-1}\left(\int_{0}^{1} 2 \hat{r} \cdot\left(\begin{array}{c}
N-1 \\
d
\end{array}\right) p_{0}(\hat{r})^{d}\right. \\
& \left.\cdot\left(1-p_{0}(\hat{r})\right)^{N-d-1} \mathrm{~d} \hat{r} \cdot\left(1-\left(1-P_{D}\right)^{d+1}\right)\right)
\end{aligned}
$$

when plugging (6) and the second equation of (10) in (14). The SU system's network false alarm probability $P_{F}^{N}\left(N ; r_{0}\right)$ can be derived by replacing $P_{D}^{C}$ with $P_{F}^{C}$ in (14).

The plots and simulation results for the detection probabilities depending on the normalized transmission range with $N=40$ are shown in Fig. 4 for various single detection probabilities. Fig. 5 shows similar plots, but now with a varying $N$ and constant $P_{D}=0.8$. Note, that the curves labeled with $N=40$ and $P_{D}=0.8$ are identical in both figures. Interpreting Fig. 5 as a three-dimensional plot with the dimensions $N, \hat{r}_{0}$ and $P_{D}^{N}$, one can derive the contour plots shown in Fig. 6. They display possible combinations of $\hat{r}_{0}$ and $N$ which yield a given network detection probability. Note, that $N$ has discrete values, resulting in plots which are not smooth. Finally, Fig. 7 shows the resulting network receiver operating characteristics $\left(\hat{r}_{0}=0.2, M=4\right.$, SNR $\left.=0\right)$ for various $N$. The dashed line represents the case for single detection as a reference. It is obvious that the detection performance increases for an increasing number of SUs in the system area. Note, that the values for $M$ and the SNR were chosen in a way, that the relative performance gain regarding the single detection case can be seen for increasing $N$. The overall performance can also be increased by using a larger $M$ (resulting in longer detection phases) or by assuming a higher SNR. 


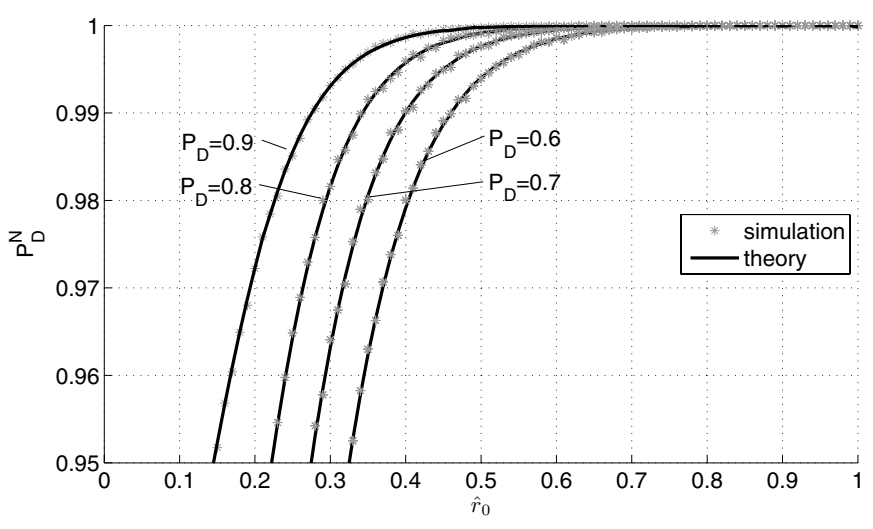

Fig. 4. Scenario with border effects: network detection probability depending on the normalized transmission range $\hat{r}_{0}$ for different single detection probabilities $P_{D}$ and $N=40$.

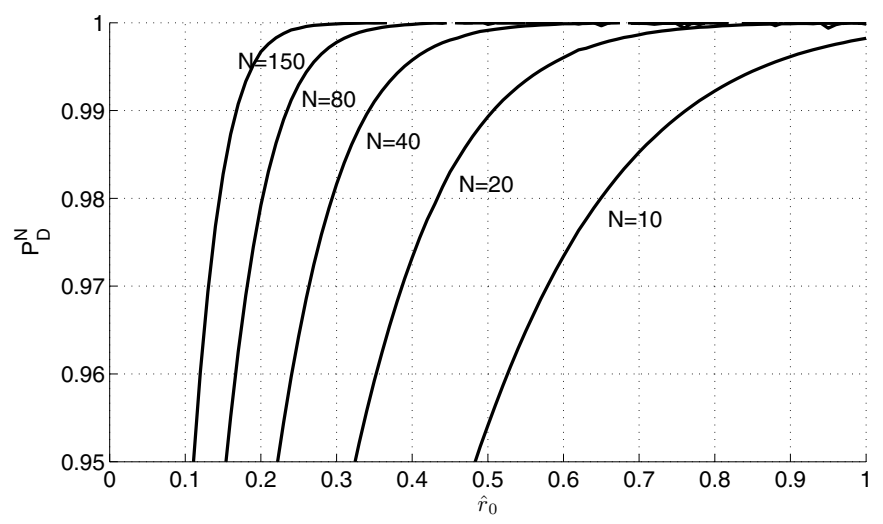

Fig. 5. Scenario with border effects: network detection probability depending on the normalized transmission range $\hat{r}_{0}$ for different numbers of SUs $N$ in the system area and $P_{D}=0.8$.

\section{CONCLusions}

Combining geometric random graph theory applied to ad hoc networks [7] with the approach of distributed detection [12] we defined the network detection and false alarm probability. A borderless scenario as well as a scenario with a bounded system area leading to border effects were investigated analytically and complemented by simulations. Additionally, the behavior of the overall receiver operating characteristic was discussed, relating the network detection probability to the network false alarm probability.

\section{REFERENCES}

[1] M. McHenry, "NSF spectrum occupancy measurements," The Shared Spectrum Company, http://www.sharedspectrum.com/?section= nsf_measurements, Tech. Rep., 2005.

[2] U. Berthold, S. Brandes, M. Schnell, and F. K. Jondral, "On Focus: OFDM Based Overlay Scenarios," in Proceedings of the 2006 IST Mobile and Wireless Communications Summit. Myconos, Greece: CDROM, June 2006.

[3] F. K. Jondral, "Cognitive radio: A communications engineering view," IEEE Wireless Commun. Mag., vol. 14, no. 4, pp. 28-33, Aug. 2007.

[4] T. Weiß and F. K. Jondral, "Spectrum pooling: an innovative strategy for the enhancement of spectrum efficiency," IEEE Commun. Mag., vol. 42, pp. 8-14, 2004.

[5] B. Bollobas, Modern graph theory. Springer, 1998.

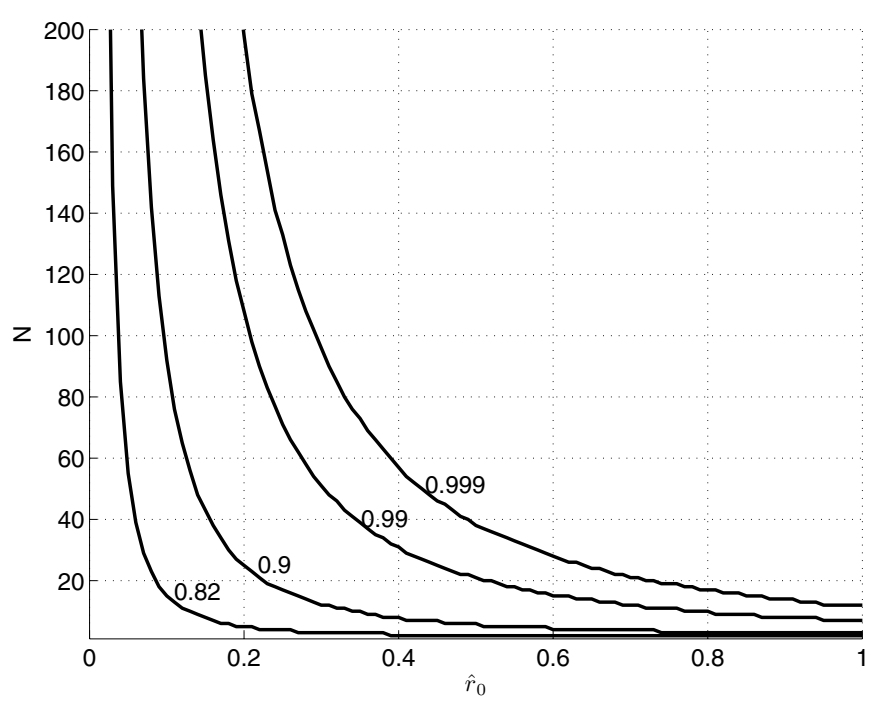

Fig. 6. Scenario with border effects: possible combinations of $N$ and $\hat{r}_{0}$ resulting in different given network detection probabilities $P_{D}^{N}\left(N ; \hat{r}_{0}\right)$.

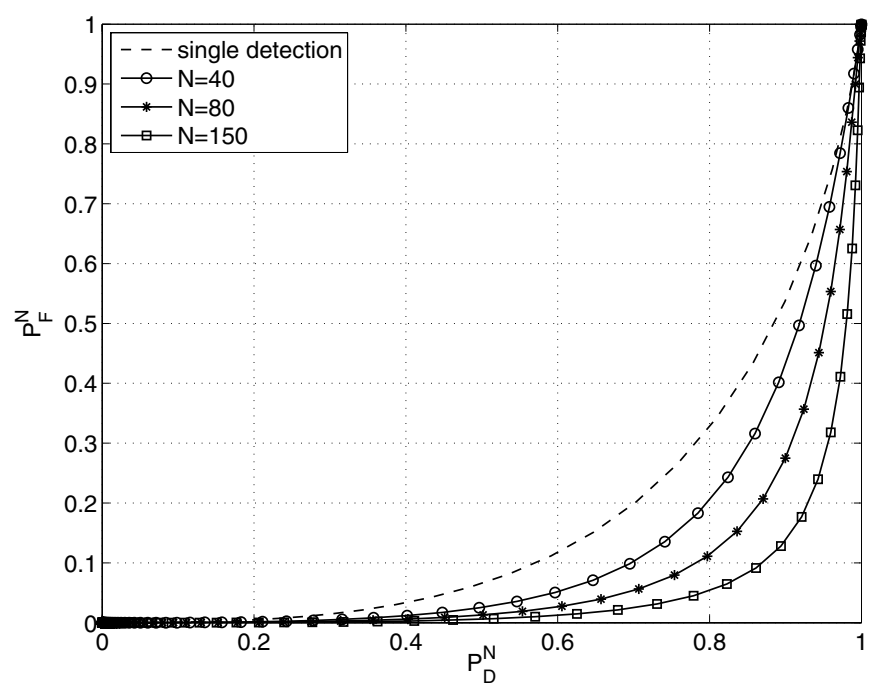

Fig. 7. Receiver operating characteristic for $\hat{r}_{0}=0.2, M=4, \mathrm{SNR}=0$.

[6] M. D. Penrose, Random geometric graphs, 1st ed. Oxford University Press, 2004.

[7] C. Bettstetter, "On the connectivity of ad hoc networks," The Computer Journal, vol. 47, no. 4, pp. 432-447, 2004.

[8] D. J. Daley and D. Vere-Jones, An introduction to the theory of point processes. Springer, 1988.

[9] J. Hillenbrand, T. Weiß, and F. K. Jondral, "Calculation of detection and false alarm probabilities in spectrum pooling systems," IEEE Commun. Lett., vol. 9, no. 4, pp. 349-351, 2005.

[10] H. Urkowitz, Signal theory and random processes. Dedham: Artech House, 1983.

[11] T. Weiß, "OFDM-basiertes Spectrum Pooling," Ph.D. dissertation, Institut für Nachrichtentechnik, Universität Karlsruhe (TH), 2004.

[12] T. Weiß, J. Hillenbrand, and F. K. Jondral, "A diversity approach for the detection of idle spectral ressources in spectrum pooling systems," in Proc. of the 48th International Scientific Colloquium, ISC 2003, Illmenau, Germany, 2003. 